\title{
ESTRUCTURA DE LA COMUNIDAD MACROFAUNÍSTICA BENTÓNICA EN LA BOCA ORIENTAL DEL ESTRECHO DE MAGALLANES, CHILE AUSTRAL.
}

\author{
BENTHIC MACROFAUNAL COMMUNITY STRUCTURE IN THE EASTERN MOUTH OF THE \\ STRAITS OF MAGELLAN, SOUTHERN CHILE.
}

Carlos Ríos ${ }^{1}$, Erika Mutschke² \& Américo Montiel ${ }^{2}$

\begin{abstract}
RESUMEN
Se describe la variabilidad en riqueza de especies y abundancia de los organismos bentónicos asociados a fondos blandos sublitorales de la entrada oriental del estrecho de Magallanes. Se identificaron un total de 49 taxa, pertenecientes a 9 categorías taxonómicas superiores. El taxon más diverso fue poliquetos con 27 taxa (56\% de los grupos macrobentónicos). Los crustáceos comprendieron 11 taxa (20\%), moluscos 4 taxa (8\%) y equinodermos 3 taxa (6\%). Los grupos cnidarios, nemertinos, priapúlidos, ascidias y braquiópodos estuvieron representados por una especie cada uno ( $10 \%$ en total). Los resultados obtenidos muestran que la comunidad bentónica se caracteriza por la presencia de una estructura de especies relativamente simple, con poblaciones numéricamente empobrecidas y una dominancia específica relativamente baja. Se observó un alto reemplazo de especies tanto entre muestras dentro de cada sector estudiado como entre los sectores de muestreo. Se sugiere que el disturbio físico causado por las corrientes del área con una consecuente disrupción de los sedimentos y una alteración significativa en los patrones de movimiento, sedimentación y erosión, sería un factor clave en el control de la composición temporal y espacial de la comunidad bentónica en el área estudiada.
\end{abstract}

Palabras clave: Subantártica, macrobentos, diversidad, riqueza de especies

\section{ABSTRACT}

This paper describes the variability in species richness and abundance of benthic organisms associated with sub littoral soft bottom at the eastern entrance of the Straits of Magellan. We identified a total of 49 taxa belonging to 9 taxonomic categories. The polychaetes were the more diverse taxon with 27 taxonomic categories (56\% of the macrobenthic groups). Crustaceans comprised 11 categories (20\%), mollusks 4 categories (8\%) and echinoderms 3 categories (6\%). The cnidarians, nemerteans, priapulids, brachiopods and ascidians were represented by one species each ( $10 \%$ in total). The results showed that the benthic community is characterized by the presence of a relatively simple structure of species with impoverished populations and relatively low specific dominance. We observed a high species turnover among samples within each sector and across studied sectors. The physical disturbance caused by the currents of the area leads to a disruption of the sediments and a significant change in movement patterns, sedimentation and erosion, and appears to be a key factor controlling the temporal and spatial composition of the benthic community in the study area.

Key words: Subantarctic, macrobenthos, diversity, species richness

\footnotetext{
${ }^{1}$ Dirección de Programas Antárticos y Subantárticos, Universidad de Magallanes. Casilla 113-D, Punta Arenas, Chile. e-mail: carlos.rios@umag.cl ${ }^{2}$ Laboratorio de Hidrobiología, Instituto de la Patagonia, Universidad de Magallanes. Casilla 113-D, Punta Arenas, Chile
} 


\section{INTRODUCCIÓN}

El estrecho de Magallanes es un ecosistema marino relativamente joven, derivado de procesos glaciares ocurridos a partir de los últimos 25.000 años (McCulloch et al. 1997). La "edad ecológica" del Estrecho es variable, dependiendo de la edad de las primeras transgresiones marinas en el área. En la zona occidental del Estrecho (océano Pacífico) las transgresiones primarias habrían ocurrido entre 14.500-13.500 años AC (Kilian et al. 2007). Las aguas del Atlántico habrían sido capaces de penetrar sobre las barras someras de la entrada oriental hace aproximadamente 9.000 años AC (McCulloch et al. 1997).

Actualmente, aguas de los océanos Atlántico, Pacífico y Austral confluyen en el Estrecho, confiriéndole una singularidad en el cono sur de Sudamérica, con patrones oceanográficos físicos diferenciales (e.g. corrientes, mareas, tasas de sedimentación) a lo largo de los ca. $500 \mathrm{~km}$ de longitud del canal (Panella et al. 1991, Antezana 1999, Antezana et al. 1992, Salinas et al. 2004, Kilian et al. 2007). Esta variabilidad ambiental ofrece una oportunidad única para el estudio de las estructuras comunitarias asociadas a diversos gradientes que ocurren a lo largo del estrecho de Magallanes (Arntz \& Ríos 1999).

Estudios sobre la estructura de comunidades marinas realizados a lo largo de gradientes ambientales existentes en la región magallánica, ya sea en conexión con determinación de patrones a escala nacional (e.g. Fernández et al. 2000, Escribano et al. 2003, Valdovinos et al. 2003), regional (e.g. Montiel et al. 2005a, 2005b, Gerdes \& Montiel 1999, Thatje \& Mutschke 1999) o local (e.g. Lorenti \& Mariani 1997, Ríos 20071), han mostrado que los valores de diversidad, biomasa y abundancia numérica son altamente heterogéneos en tiempo y espacio. La alta heterogeneidad en factores ambientales existentes en los sistemas marinos de Magallanes, entre ellos la presencia/ausencia de glaciares, las velocidades de corrientes y mareas, el tipo de sedimentos y las tasas de disturbios físicos parecen ser parámetros relevantes en la determinación de la distribución y abundancia de los organismos bentónicos (Gerdes \& Montiel 1999, Gutt et al. 1999, Ríos 20071). La alta variabilidad ambiental en este tipo de sistemas y sus efectos en la distribución de especies, estructura comunitaria y diferencias en comunidades también ha sido observada en fiordos y canales noruegos (e.g. Holte 1998; Oug 2000). Recientemente, Ríos $(2007)^{1}$ ha mostrado que una característica común de algunas comunidades marinas en Magallanes es la mantención de niveles de riqueza de especies relativamente altos, sugiriendo que ello sería consecuencia de una alta tasa de reemplazo de especies tanto en el tiempo como en el espacio (Ríos et al. 2003, Ríos et al. 2005).

En el presente trabajo se entrega un análisis de la estructura de la comunidad faunística y su relación con las características granulométricas de los sedimentos, a partir de muestras cuantitativas tomadas en el sublitoral de la boca atlántica del estrecho de Magallanes. Los objetivos del trabajo fueron caracterizar la comunidad macrobentónica sobre la base de su composición específica, abundancia numérica, riqueza y diversidad de especies y sus preferencias de hábitat (e.g. estructura del sedimento).

\section{MATERIAL Y MÉTODOS \\ Área de estudio}

El área de estudio comprende específicamente la entrada somera atlántica del estrecho de Magallanes (Fig. 1). Este sector del Estrecho presenta profundidades que varian entre 30-50 m. Las aguas del sector son originadas a partir de la mezcla de la capa superficial de las aguas intermedias del océano Atlántico provenientes del sur, con aguas más diluidas en términos de salinidad y de origen continental que fluyen desde el estrecho de Magallanes hacia la plataforma continental Atlántica (Glorioso 1987). La columna de agua es verticalmente homogénea como resultado de la mezcla significativa originada por las fuertes corrientes mareales presentes en el área.

Hidrodinámicamente, el área de estudio se caracteriza por fuertes corrientes mareales que se originan a partir de las olas de mareas semi-diurnas en el océano Atlántico y que determinan un régimen de mareas macromareales, con una amplitud de $8 \mathrm{~m}$ durante las mareas de la primavera austral (Medeiros \& Kjerfve 1988). En la boca atlántica y a una profundidad de $40 \mathrm{~m}$, se han registrado velocidades de corrientes entre $80-100 \mathrm{~cm} / \mathrm{s}$ con una dirección este-oeste, la cual en el fondo se puede reducir a la mitad debido a la fricción (Michelato et al. 1991). Las fuertes corrientes y las profundidades relativamente someras del área resultan en un sistema hidrodinámico de elevada energía, con transporte de gravas y arenas, zonas de erosión y un sustrato con predominancia de gravas, gravas arenosas y arenas gravosas (Brambati et al. 1991).

Durante el período de estudio (junio de 2003),

${ }^{1}$ Ríos, C. 2007. Marine benthic communities of the Magellan Region, Southern Chile: contribution of different habitats to the overall biodiversity. $\mathrm{PhD}$ Thesis, Alfred Wegener Institut for Polar and Marine Research-Bremen University, Germany. 122 pp. Disponible en http://nbn-resolving. de/urn:nbn:de:gbv:46-diss000110638 
la columna de agua presentó una salinidad promedio que varió entre 31-32 PSU, una temperatura media entre $4,2-4,5^{\circ} \mathrm{C}$ y valores de oxígeno disuelto entre 8-14 $\mathrm{ml} / \mathrm{L}$.

\section{Recolección de la información}

El muestreo fue realizado entre el 25 y 27 de junio de 2003 (invierno austral). Entre el extremo de la isla de Tierra del Fuego (punta Catalina) y el sector de bahía Posesión por el continente (punta Daniel) se delimitaron 3 áreas de muestreo de $500 \mathrm{~m}^{2}$ cada una (Fig. 1), cubriendo la porción central (CT) y las aledañas a Tierra del Fuego (PC) y al continente (PD). La ubicación de las tres áreas estuvo determinada fundamentalmente por la existencia de plataformas de explotación de hidrocarburos en el subsuelo marino y por el track de navegación por donde ingresan y salen embarcaciones hacia y desde el estrecho de Magallanes. La profundidad promedio en el sector de punta Catalina fue $16,1 \mathrm{~m}$ (de=3,2; $=14)$, en el sector central $61,1 \mathrm{~m}$ (de=1,74; $\mathrm{n}=14)$ y $21,8 \mathrm{~m}(\mathrm{de}=1,4 ; \mathrm{n}=14)$ en el sector de punta Daniel.

En cada una de las tres áreas se determinaron aleatoriamente 14 estaciones para el muestreo de sedimentos, los cuales fueron recolectados con una draga Smith-McIntyre de 0,1 $\mathrm{m}^{2}$ ) desplegada desde el L/C "Luma". En cada punto de muestreo se tomaron tres réplicas para la separación de la fauna y una muestra adicional para el análisis granulométrico. Cada punto de muestreo fue georreferenciado mediante GPS, utilizando el datum Provisional South Chilean 1963 Chile (Hito XVIII).

Con el objeto de separar la fracción macrobentónica de las muestras de sedimentos, éstas fueron cernidas a través de tamices con una abertura de malla de $0,5 \mathrm{~mm}$. Los organismos separados fueron contabilizados e identificados hasta el nivel taxonómico más bajo posible.

Para la caracterización granulométrica de cada estación de muestreo, se lavó una fracción de aproximadamente $500 \mathrm{~g}$ en agua, agregándose el material decantado a la muestra original. La muestra, incluyendo el material de lavado, se secó en un horno eléctrico a $96^{\circ} \mathrm{C}$ durante $24 \mathrm{hrs}$. El material seco se tamizó en un vibrador eléctrico Podmore ${ }^{\circledR}$, utilizando una batería de 6 tamices $(3,35 \mathrm{~mm}, 2 \mathrm{~mm}, 1 \mathrm{~mm}$, $500 \mu \mathrm{m}, 250 \mu \mathrm{m}, 125 \mu \mathrm{m}, 63 \mu \mathrm{m}$ y una bandeja para los sedimentos finos). El material grueso retenido en el primer tamiz $(3,35 \mathrm{~mm})$, fue estimado medi- ante el tamaño medio de las gravas. Los tamaños de los sedimentos se transformaron desde unidades métricas a unidades adimensionales $\phi$, mediante la fórmula $\phi=-\log _{2}\left(d / d_{0}\right)=\left(-\log _{10} d\right) /\left(\log _{10} 2\right)$, donde $d$ es el diámetro expresado en $\mathrm{mm}$ y $d_{0}$, es el diámetro de un grano de $1 \mathrm{~mm}$. Los parámetros estadísticos (media, sigma, asimetría y curtosis) se calcularon con el método gráfico tradicional (Folk 1974), a partir de los tamaños ø5, ø16, ø25, ø50, ø75, ø84 y ø90. Las muestras se clasificaron en clases texturales utilizando el diagrama de Shepard (1954).

\section{Análisis de la información}

Todos los análisis faunísticos fueron realizados con el programa computacional PRIMER v6 (Clarke \& Gorley 2006). Sobre la base de la composición cuantitativa de la macrofauna y dependiente de los resultados de análisis multivariados de ordenación y agrupamiento, se calcularon diversos índices ecológicos univariados: número de especies $(\mathrm{S})$, abundancia $(\mathrm{N})$ o densidad expresada como número de individuos $/ \mathrm{m}^{2}$, diversidad comunitaria utilizando el índice de Shannon-Wiener (H', transformación logarítmica natural, Magurran 2004), uniformidad de la distribución de individuos entre las especies (J, Magurran 2004) y la diversidad de especies según Margalef (d, Magurran 2004). Para evaluar la similitud/disimilitud en la composición de la comunidad entre las áreas de muestreo, se aplicaron las técnicas multivariadas de agrupamiento ("cluster analysis") y escalamiento multidimensional (MDS) con los datos transformados a la raíz cuarta, previo a la aplicación del índice de similitud de Bray-Curtis (Bray \& Curtis 1957; véase la discusión de Yoshioka 2008a, 2008 b y Somerfield 2008 respecto de la aplicabilidad de tal índice a este tipo de análisis). La significancia estadística de las agrupaciones obtenidas con el análisis de agrupamiento se evaluó con la rutina SIMPROF ("similarity profile". La comparación de la composición específica entre los tres sitios de muestreo se realizó con la rutina ANOSIM ("analysis of similarities"), bajo la hipótesis nula de que no hay diferencias en la composición macrofaunística en estos tres sectores. La contribución de las especies/taxa a la diferenciación o similitud entre grupos se analizó con el modulo SIMPER ("similarity percentages"). Las correlaciones entre los parámetros comunitarios (abundancia y diversidad) y los factores ambientales (profundidad, porcentajes de grava, arena y limos-arcillas) fueron estimadas a través del coeficiente de correlación 
Spearman (Rho) con el respectivo cálculo de su significancia estadística. Previo al análisis, todos los valores porcentuales fueron transformados utilizando arcoseno (Sokal \& Rohlf 2003). Estos análisis fueron realizados con el programa StatView v5.

Las comparaciones de promedios fueron realizadas mediante análisis de t y de varianza (ANOVA) de una vía según corresponda, mientras que para las comparaciones post-hoc de los promedios se utilizó el método HSD de Tukey (Sokal \& Rohlf 2003).

\section{RESULTADOS}

Características granulométricas del sustrato

El tamaño promedio de los sedimentos para las 42 muestras analizadas fue de $-0,036 \phi$, con una desviación estándar promedio de 1,86申, una asimetría de 0,005 y una curtosis de 1,17. Estos resultados indican que el tamaño promedio de los sedimentos presentes en el área de estudio corresponde a arenas gruesas de $1 \mathrm{~mm}$. El análisis textural indicó la existencia de las siguientes clases de sedimentos: $\mathrm{B}$ (barro), BA (barro arenoso), A (arena), AG (arena gravosa), GA (grava arenosa). En el sector costero de la isla (punta Catalina), los sedimentos predominantes fueron arenas con ausencia relativa de gravas.

La desviación estándar $(\sigma)$, alcanzó un valor promedio de 1,8 $\phi$ para las tres estaciones sublitorales. En general, existe una tendencia en las áreas de muestreo a presentar desde arenas finas en la porción continental, con valores que no superan a $2 \phi$; arenas gruesas en el centro del estrecho y muy gruesas (arena-grava), en el extremo insular. La asimetría no mostró ninguna tendencia espacial entre los 3 sectores, indicando una mayor ausencia de sedimentos finos, probablemente debido a procesos de depositación de la zona submareal. Los escasos valores positivos de asimetría fueron calculados en varios casos en zonas más profundas (e.g. centro del Estrecho), donde presumiblemente hay menor flujo y predominan procesos de depositación de sedimentos finos por sobre procesos erosivos.

La distribución espacial de la textura predominante de los sedimentos se indica esquemáticamente en la figura 2. El área de muestreo puede ser dividida en tres zonas texturales: a) ambiente sublitoral continental, b) ambiente sublitoral medio y c) ambiente sublitoral isla. Los parámetros estadísticos correspondientes se presentan en la tabla 1.

El ambiente sublitoral central se subdividió en dos áreas para caracterizar óptimamente la distribución textural, ya que ésta presenta dos clases de textura muy distintas; la primera, con un promedio de $-1,19 \phi$, que representa gravillas y la segunda con un promedio de $3 \phi$, que representa arenas muy finas. El ambiente sublitoral continental presentó un promedio de 0,65 $\phi$, que representa arenas gruesas y el insular, un promedio $\mathrm{de}-1,65 \phi$ que representa arenas muy gruesas.

\section{Caracterización del macrobentos}

En las muestras recolectadas, provenientes de las tres áreas de muestreo se identificó un total de 49 taxa, pertenecientes a 9 categorías taxonómicas superiores (Tabla 2). El taxon más diverso fue poliquetos con 27 taxa (56\% de los grupos macrobentónicos). Los crustáceos comprendieron 11 taxa (20\%), moluscos 4 taxa (8\%) y equinodermos 3 taxa (6\%). Los grupos cnidarios, nemertinos, priapúlidos, ascidias y braquiópodos estuvieron representados por una especie cada uno ( $10 \%$ en total). Las especies que presentaron las mayores frecuencias de ocurrencia fueron los poliquetos Kinbergonuphis dorsalis (presente en el 7\% de las muestras) y Scoloplos (Leodamas) ohlini (4\%) y el equinodermo Athyonidium chilensis (5\%).

La abundancia total del macrobentos por estación de muestreo varió entre 3,3 y $56 \mathrm{ind} / \mathrm{m}^{2}$, con un promedio de $18,5 \mathrm{ind} / \mathrm{m}^{2}$ (de=18,48; $\mathrm{N}=42$ ). El área con mayor densidad fue punta Catalina ( $\mathrm{x}=26,7$ ind $/ \mathrm{m}^{2}$; de $=13,1 ; \mathrm{N}=42$ ), mientras que la menor fue determinada para punta Daniel $\left(\mathrm{x}=9,5 \mathrm{ind} / \mathrm{m}^{2} ; \mathrm{ds}=5,5\right.$; $\mathrm{N}=42$ ) (Fig. 3). Las densidades promedios por área de muestreo resultaron ser significativamente diferentes (ANOVA; $\left.\mathrm{F}^{(2,42)}=9,693 ; \mathrm{p}=0,0004\right)$. De acuerdo a la prueba HSD de Tukey, el sitio próximo al continente (punta Daniel) difiere significativamente de los dos restantes $(p<0,05)$, mientras que las densidades obtenidas para punta Catalina y el sector central del Estrecho no fueron significativamente diferentes ( $>00,05)$.

En las tres áreas de muestreo la abundancia macrobentónica estuvo dominada por los poliquetos, mientras que los restantes grupos dominantes dependieron del área estudiada (Fig. 4). Los equinodermos (e.g. Athyonidium chilensis), crustáceos (e.g. Cirolana albinota) y sipuncúlidos (e.g. Themiste sp.), predominaron en el sector de punta Catalina. En el área central del Estrecho predominaron los sipuncúlidos (Themiste sp.), crustáceos (isópodos en general) y moluscos (principalmente Mytilus chilensis), mientras que en el área de punta Daniel hubo un predominio de crustáceos (e.g. Serolis convexa), equinodermos (Athyonidium 
chilensis) y moluscos (e.g. Mactra cf. patagonica).

La riqueza de especies varió entre las áreas estudiadas, con un total de 19 taxa identificadas en las muestras del área de punta Daniel (1-3 taxa/ muestra), 31 en las muestras del sector central (1-5 taxa/muestra) y 34 en las muestras de punta Catalina (1-6 taxa/muestra). El 30\% de las muestras colectadas en las tres áreas de muestreo $(n=40)$ no presentaron evidencias de macrofauna.

\section{Ensambles de especies y estructura comunitaria}

El análisis de conglomerados basado en las abundancias promedios por especies no mostró evidencias de agrupación de las estaciones de muestreo en cada área de estudio (Fig. 5). Según los valores obtenidos con SIMPROF, en ninguno de los tres casos existen evidencias de agrupaciones genuinas de las muestras involucradas, i.e. las muestras difieren significativamente entre sí, en términos de su estructura (especies y abundancias).

La variabilidad observada en riqueza de especies, abundancias y tipos de especies o taxa dominantes entre los tres sectores de muestreo se expresó también en el análisis multidimensional y de agrupación realizado, considerando los valores promedios de abundancia para los sectores (Fig. 6A y B). En el dendrograma (Fig. 6A) no se observa ninguna estructura definible tanto dentro como entre los sitios de muestreo. Sin embargo, los resultados obtenidos para punta Daniel (PD en la figura) tienden a conformar una estructura relativamente homogénea en términos de tipos de especies/taxa y sus correspondientes abundancias, en comparación con las agrupaciones obtenidas para punta Catalina (PC) y sector central del estrecho (CT). Esta situación se corroboró parcialmente con los resultados obtenidos del ordenamiento multidimensional (Fig. $6 \mathrm{~B})$, en donde se aprecia una separación del conjunto de especies recolectadas en el área de punta Daniel, en comparación con la ordenación para las áreas de punta Catalina y sector central del Estrecho.

Los resultados obtenidos con ANOSIM indican que la hipótesis de no diferencia en la composición macrofaunística entre las tres localidades estudiadas debe ser rechazada $(R=0,3 ; p<0,01)$. Según la comparación pareada entre las tres áreas, las composiciones macrofaunísticas determinadas para punta Catalina y sector central de Estrecho no resultaron ser significativamente diferentes $(R=0,2 ; p=0,04)$, mientras que punta Daniel difiere estadísticamente de punta Catalina
$(R=0,3 ; p<0,01)$ y del sector central $(R=0,3 ; p<0,01)$.

Tomando en cuenta los resultados obtenidos con ANOSIM y las diferencias significativas encontradas respecto de las densidades medias y del número promedio de especies/taxa superiores, los parámetros univariados relacionados con la estructura comunitaria se calcularon considerando en conjunto los valores para punta Catalina y sector central y en forma separada los de punta Daniel (Tabla 3). Los valores de diversidad medida con los índices de Margalef (d) y de ShannonWiener $\left(\mathrm{H}^{\prime}\right)$ resultaron ser significativamente mayores en el grupo de muestras formado por las áreas de punta Catalina y el sector central del Estrecho, en comparación con los valores obtenidos para punta Daniel $(\mathrm{t}=3,826$; $p=0,0007$ y $t=2,89 ; p=0,005$, respectivamente). Los valores de uniformidad $(\mathrm{J})$ fueron similares en ambos grupos ( $t=1,024 ; p=0,281)$.

Los resultados obtenidos con la aplicación del análisis SIMPER para determinar la contribución de especies en las agrupaciones de muestras se entregan en la tabla 4. Diferentes especies de poliquetos son las que contribuyeron de manera más significativa tanto a la similitud dentro de cada grupo como en la disimilaridad entre ellos, especialmente en las áreas de punta Catalina y centro del Estrecho. En el grupo conformado por estas áreas, tres especies de poliquetos y una de sipuncúlido contribuyeron con más del $50 \%$ a la similitud dentro del grupo. En punta Daniel existió una mayor heterogeneidad taxonómica, aunque sólo una especie de poliqueto generó más del $55 \%$ de la similitud dentro del grupo. Diez especies contribuyeron con más del $50 \%$ de la disimilitud entre los grupos analizados, de las cuales 8 fueron poliquetos además de un holothuroideo (Athyonidium chilensis) y un sipuncúlido (Themiste sp.).

En cuanto a las relaciones entre las características comunitarias y las variables abióticas, los resultados indican que la abundancia, riqueza de especies y diversidad presentan una relación significativa con el porcentaje de grava y limo. Sin embargo, no se encontró ninguna relación entre las características comunitarias y la profundidad (Tabla 5).

\section{DISCUSIÓN}

Los resultados obtenidos en este estudio indican que el componente bentónico sublitoral de la boca oriental (entrada Atlántica) del estrecho de Magallanes se caracteriza por la presencia de una estructura de especies (riqueza) relativamente simple, 
con poblaciones numéricamente empobrecidas y una dominancia específica relativamente baja. Asimismo, se observó un alto reemplazo de especies tanto entre muestras dentro de cada sector estudiado como entre sectores de muestreo. Esta característica indica una alta heterogeneidad biótica del área, asociada a fuertes procesos de disturbios ambientales capaces de generar, asimismo, una gran heterogeneidad ambiental del sector. Características similares han sido encontradas para otros sectores estudiados en el sublitoral del Estrecho (Ríos 2007) 1 .

En relación con la riqueza de especies macrobentónicas determinada para el área, los resultados de este estudio concuerdan bien con un probable gradiente (incremento en riqueza de especies hacia el sector Pacífico del Estrecho) sugerido en otros estudios realizados en el área (e.g. Montiel et al. 2001, Ríos et al. 2005, Mutschke \& Ríos 2006).

Para el sector comprendido entre la primera y segunda Angosturas del estrecho de Magallanes, Ríos et al. (2003) reportaron un total de 301 taxa recolectados en seis períodos diferentes de muestreos, con un alto reemplazo de especies entre períodos de muestreo y también entre muestras por período. La riqueza específica en este sector del Estrecho fue marcadamente superior a la determinada en el presente trabajo para la boca oriental. Pizarro $(2008)^{2}$, en su estudio realizado a inicios de la primavera austral y a lo largo de un gradiente ambiental entre áreas aún bajo la influencia de glaciares (Campo Nevado; sector Pacífico del Estrecho) y áreas actualmente deglaciadas (Paso Ancho, sector central del Estrecho), determinó la presencia de 64 taxa pertenecientes a 20 familias de invertebrados. El gradiente en disminución de riqueza de especies hacia la entada oriental del Estrecho también se ha observado en otros componentes (e.g. zooplancton) del sistema marino del estrecho de Magallanes (Mazzochi \& Ianora 1991).

El grado de cambio en la composición de especies entre diferentes ensambles a lo largo del estrecho de Magallanes es relativamente alto. Sólo 18 taxa de los 49 registrados en este estudio fueron registrados en el estudio realizado por Ríos et al. (2003) en el segundo bolsón del Estrecho (6 poliquetos, 5 crustáceos, 1 equinodermo, 1 holoturoideo, 1 braquiópodo, 1 sipuncúlido y 3 moluscos). En relación con otros resultados obtenidos para comunidades sublitorales de la región magallánica - aunque con otras técnicas de muestreo- el ensamble identificado para la entrada oriental del Estrecho comparte sólo 5 especies ( 3 poliquetos, 1 crustáceo y 1 braquiópodo) con el conjunto registrado para bahía San Sebastián (Tierra del Fuego, costa Atlántica) por López-Gappa \& Sueiro 2007). En este sector se identificaron un total de 113 taxa de invertebrados macrobentónicos. En relación con el taxa dominante en este tipo de ambientes sublitorales, sólo seis géneros de las 26 especies de poliquetos identificadas en el presente estudio son compartidos con la poliquetofauna identificada por Montiel et al. (2007) para las costas noroccidental y sur de Tierra del Fuego.

La heterogeneidad en la composición específica de los tres sectores estudiados se reflejó en la distinción de dos ensambles relacionados con distintas porciones sublitorales de la entrada oriental del Estrecho. Esta distinción estuvo en concordancia con las características granulométricas de los sedimentos del área, los cuales, en general, se caracterizaron por la predominancia de arenas muy gruesas y la ausencia de sedimentos finos con una baja acumulación de material orgánico en el fondo, debido al constante movimiento de arena y gravillas. El sedimento presente en esta zona es afectado por los disturbios que ocasionan las fuertes corrientes marinas lo que, unido a las someras profundidades existentes en la cuenca, resultan en el transporte de gravas y arenas, aguas turbulentas y zonas de erosión (Brambati et al. 1991).. Las corrientes mareales resultantes del ingreso de masas de aguas del océano Atlántico en este sector del Estrecho pueden alcanzar velocidades de $2,5 \mathrm{~m} / \mathrm{seg}$, con amplitudes mareales de $9 \mathrm{~m}$ (Brambati et al. 1991, Medeiros \& Kjerfve 1988). En el sector costero de Tierra del Fuego, los sedimentos predominantes fueron arenas con ausencia relativa de gravas, indicando niveles de energía de flujo moderados, por lo que se estima que estas zonas quedan fuera de la corriente principal del Estrecho.

Consecuentemente, el disturbio físico causado por las corrientes del área y que conduce a una disrupción de los sedimentos y a una alteración significativa en los patrones de movimiento, sedimentación y erosión, parece ser un factor clave en el control de la composición temporal y espacial de la comunidad bentónica. El efecto de las corrientes también ha sido sugerido como un factor crítico para definir diferencias en la composición de especies de otros componentes del sistema a lo largo del estrecho (e.g. fitoplancton, Uribe 1991). Las características estructurales de la comunidad estudiada sugieren la existencia de un

${ }^{2}$ Pizarro, J. 2008. Estructura comunitaria de la macrofauna bentónica del estrecho de Magallanes y su posible aplicación en la Bioseguridad. Tesis presentada para optar al Título Profesional de Biólogo Marino.

Departamento de Ciencias y Recursos Naturales, Facultad de Ciencias, Universidad de Magallanes. 104 pp. 
ambiente inestable, sometido a disturbios físicos constantes causados por las fuertes corrientes y que determinarían bajas abundancias y una alta diversidad beta, aunque con una riqueza de especies más bien restringida, en comparación con otras secciones del estrecho. Estas características ambientales pueden generar cambios en los atributos taxonómicos (tipo de especies) y funcionales (tipos de alimentación) de las comunidades, como ha sido demostrado por ejemplo para comunidades de nemátodos, por Schratzberger et al. (2009).

Los resultados obtenidos hasta la fecha muestran la complejidad para definir un modelo de "comunidad típica" para los sistemas marinos de la región de Magallanes, como por ejemplo sugieren LópezGappa \& Sueiro (2007). La región magallánica es un dominio altamente heterogéneo desde un punto de vista ambiental y expuesto a regímenes de disturbios totalmente diferentes según sean los sectores que se consideren: Esta heterogeneidad en las características del ambiente parecen generar una importante diversidad temporal y espacial de los ensambles de macroorganismos bentónicos presentes en la región de Magallanes.

TABLA 1. Parámetros estadísticos asociados a las clases granulométricas presentes en los sedimentos de tres áreas sublitorales en el sector oriental del estrecho de Magallanes.

\begin{tabular}{lcccc}
\hline Ambiente & Tamaño medio $(\phi)$ & Desviación estándar $(\phi)$ & Skewness & Curtosis \\
\hline a) Sublitoral Punta Daniel & 0,65 & 0,78 & $-0,05$ & 1,28 \\
b) Sublitoral centro 1 & $-1,19$ & 2,31 & $-0,21$ & 0,87 \\
b) Sublitoral centro 2 & 3,00 & 2,14 & $-0,7$ & 2,97 \\
c) Sublitoral Punta Catalina & $-1,65$ & 2,55 & 0,4 & 0,82 \\
\hline
\end{tabular}

TABLA 2. Listado taxonómico de los macroorganismos bentónicos sublitorales recolectados con una draga Smith-McIntyre en el sector oriental del estrecho de Magallanes.

\section{CNIDARIA \\ Cnidaria Indet. \\ NEMERTINI \\ Nemertini Indet. \\ SIPUNCULIDA \\ Themiste sp. \\ MOLLUSCA}

Photinula caerulescens King, 1831

Mytilus chilensis (Hupé, 1854)

Mactra cf. patagonica

Eurhomalea exalbida (Chemnitz, 1795)

POLYCHAETA

Aglaophamus polyphara (Schmarda, 1861)

Aglaophamus cf. peruana

Aglaophamus virginis (Kinberg, 1866)

Nephtys imbricata Grube, 1857

Nephtys sp.

Ninoe leptognatha Ehlers, 1900

Lumbrineris cingulata (Ehlers, 1897)

Notocirrus lorum Ehlers, 1897

Maldanidae Indet.

Euclymene grossa (Baird, 1871)

Glycinde armata (Baird, 1871)

Hemipodus heteropapillatus Hartmann-Schröder,

1962

Eteone sculpta Ehlers, 1897

Eteone aurantiaca Schmarda, 1861

Onuphidae Indet.

Kinbergonuphis dorsalis (Ehlers, 1897)

Scoloplos (Leodamas) ohlini (Ehlers, 1900)

Scolelepis sp.

Boccardia wellingtonensis Rood, 1975
Polynoidae Indet

Harmothoe sp.

Terebellidae Indet.

Thelepus setosus (De Quatrefages, 1866)

Gymnonereis hartmannschroederae Pettibone, 1970

Ampharete sp.

Capitellidae Indet.

Pilargidae Indet.

CRUSTACEA

Gammaridae Indet.

Munna sp.

Cirolana albinota Vanhöffen, 1914

Cristaserolis convexa (Cunningham, 1871)

Acanthoserolis schythei (Lütken, 1858)

Neastacilla magellanica (Ohlin, 1901)

Antarcturus americanus Beddard,1886

Edotea sp.

Edotea transversa Menzies, 1942

Peltarion spinosulum (White, 1843)

ECHINODERMATA

Anasterias antarctica (Lütke, 1857)

Amphiura magellanica Ljungemon, 1867

Athyonidium chilensis (Semper, 1868)

UROCHORDATA

Ascidea Indet.

BRACHIOPODA

Magellania venosa (Solander, 1786) 
TABLA 3. Mediciones univariadas de la estructura comunitaria para en el sector oriental del estrecho de Magallanes, calculadas sobre la base de abundancias estandarizados a $1 / \mathrm{m}^{2}$. Se incluye el número total de especies $(\mathrm{S})$ y de individuos (N), diversidad según Margalef (d), uniformidad según Pielou (J) y diversidad según Shannon-Wiener (H'; en base e). PC+CTR = punta Catalina y Centro en conjunto; PD = punta Daniel (PD).

\begin{tabular}{cccccc}
\hline & $\mathrm{S}$ & $\mathrm{N}$ & $\mathrm{d}$ & $\mathrm{J}$ & $\mathrm{H}^{\prime}$ \\
\hline $\mathrm{PC}+\mathrm{CTR}$ & 47 & 1940 & 6,08 & 0,87 & 3,35 \\
PD & 19 & 420 & 2,98 & 0,89 & 2,64 \\
\hline
\end{tabular}

TABLA 4. Especies características que definen tanto la similitud dentro de grupos de sitios de muestreo como la disimilitud entre ellos $(\mathrm{PC}+\mathrm{C}=$ punta Catalina y Centro en conjunto; $\mathrm{PD}=$ punta Daniel). Resultados obtenidos con la rutina SIMPER de PRIMER.

$\mathrm{AP}=$ abundancia promedio; $\mathrm{SP}=$ similitud promedio; $\mathrm{Sim}=$ similitud; $\mathrm{DS}=$ desviación estándar; $\mathrm{CTB}=$ contribución porcentual; $\mathrm{ACM}=$ porcentaje acumulativo; $\mathrm{DIP}=$ disimilitud promedio; Dis=disimilitud.

\begin{tabular}{lcccccc}
\hline \multicolumn{1}{c}{ SIMILITUD } & & & & & & \\
GRUPO PC+C & AP & SP & SimDS & & CTB\% & ACM\% \\
\hline Kinbergonuphis dorsalis & 0,70 & 4,29 & 0,52 & & 26,35 & 26,35 \\
Scoloplos (Leodamas) ohlini & 0,44 & 2,02 & 0,26 & & 12,36 & 38,72 \\
Themiste sp. & 0,49 & 1,55 & 0,33 & & 9,51 & 48,23 \\
Euclymene grossa & 0,38 & 1,18 & 0,24 & & 7,27 & 55,49 \\
& & & & & & \\
GRUPO PD & & & & & & \\
Nephtys imbricata & 0,77 & 11,53 & 0,64 & & 56,85 & 56,85 \\
Athyonidium chilensis & 0,61 & 4,83 & 0,43 & & 23,81 & 80,66 \\
Cristaserolis convexa & 0,29 & 1,01 & 0,18 & & 5,00 & 85,66 \\
\hline$\quad$ DISIMILITUD & & & & & & \\
$\quad$ GRUPOS PC+C \& PD & AP & AP & DIP & Dis/DS & CTB\% & ACM\% \\
\hline Nephtys imbricata & 0,28 & 0,77 & 7,85 & 0,93 & 8,63 & 8,63 \\
Kinbergonuphis dorsalis & 0,70 & 0,10 & 6,51 & 0,90 & 7,16 & 15,80 \\
Athyonidium chilensis & 0,35 & 0,61 & 6,43 & 0,86 & 7,07 & 22,87 \\
Scoloplos (Leodamas) ohlini & 0,44 & 0,11 & 5,45 & 0,58 & 5,99 & 28,86 \\
Aglaophamus virginis & 0,32 & 0,31 & 4,49 & 0,65 & 4,94 & 33,81 \\
Themiste sp. & 0,49 & 0,00 & 3,91 & 0,67 & 4,31 & 38,11 \\
Euclymene grossa & 0,38 & 0,00 & 3,64 & 0,53 & 4,00 & 42,11 \\
Glycinde armata & 0,39 & 0,10 & 3,55 & 0,62 & 3,90 & 46,01 \\
Eteone aurantiaca & 0,23 & 0,19 & 3,38 & 0,54 & 3,72 & 49,74 \\
Ampharete sp. & 0,33 & 0,10 & 3,33 & 0,58 & 3,67 & 53,40 \\
\hline
\end{tabular}

TABLA 5. Valores de Spearman (Rho) para la correlación entre la abundancia, riqueza de especies y diversidad (H') versus las variables abióticas. En negritas los valores significativos $(\mathrm{P}<0.05)$.

\begin{tabular}{ccccc}
\hline Especies & PROF & $\begin{array}{c}\text { Grava } \\
(\%)\end{array}$ & $\begin{array}{c}\text { Arena } \\
(\%)\end{array}$ & Limo (\%) \\
\hline Promedio Riqueza de especies & 0,008 & 0,5 & $-0,5$ & 0,4 \\
Promedio Abundancia (ind $\left./ \mathrm{m}^{2}\right)$ & 0,005 & 0,6 & $-0,5$ & 0,5 \\
Diversidad (H') & 0,05 & 0,5 & $-0,4$ & 0.5 \\
\hline
\end{tabular}




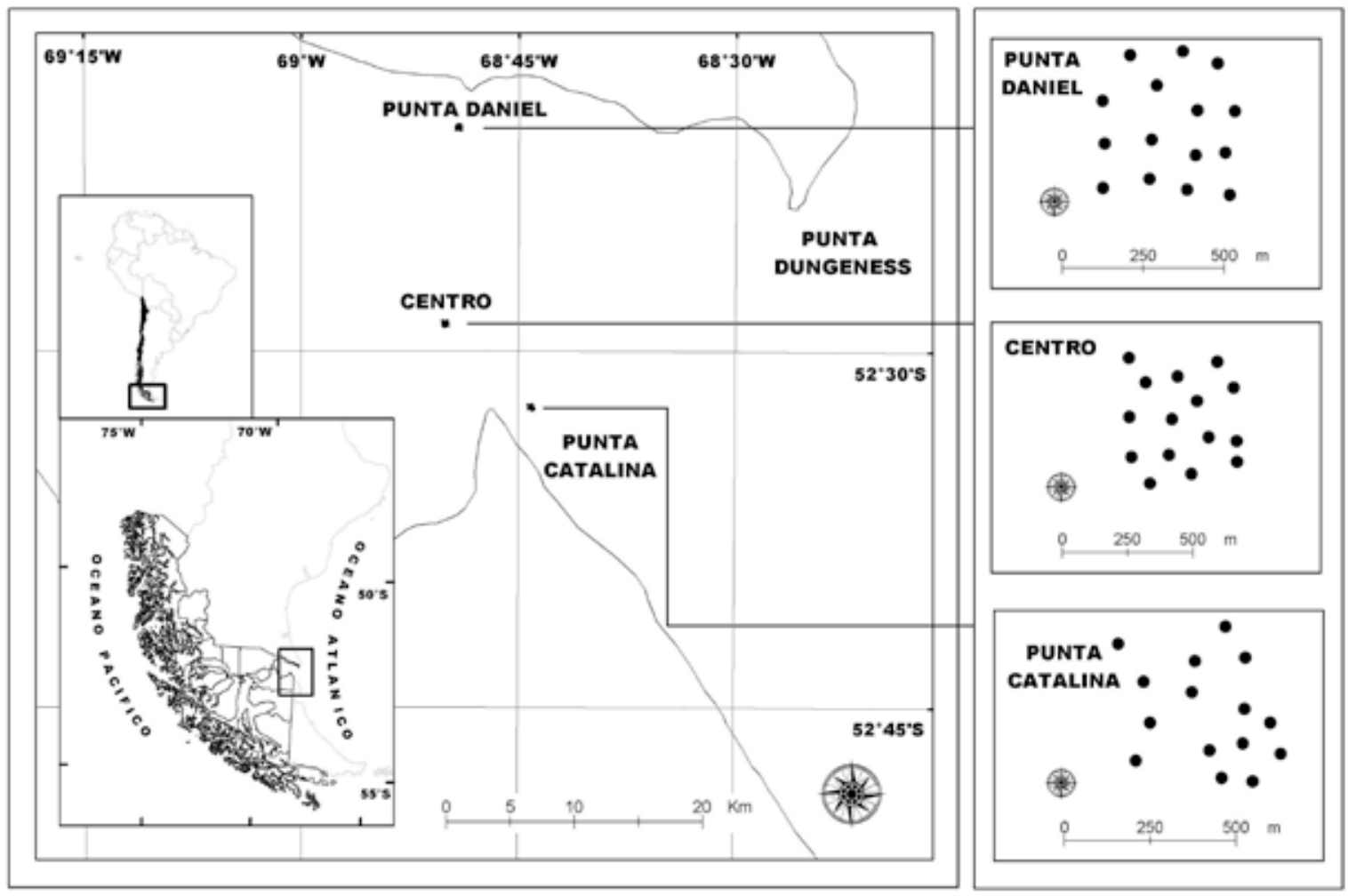

Fig. 1. Áreas de estudio en la entrada oriental (Atlántica) del estrecho de Magallanes. Los puntos indicados para cada área en particular representan los sitios específicos de muestreo.
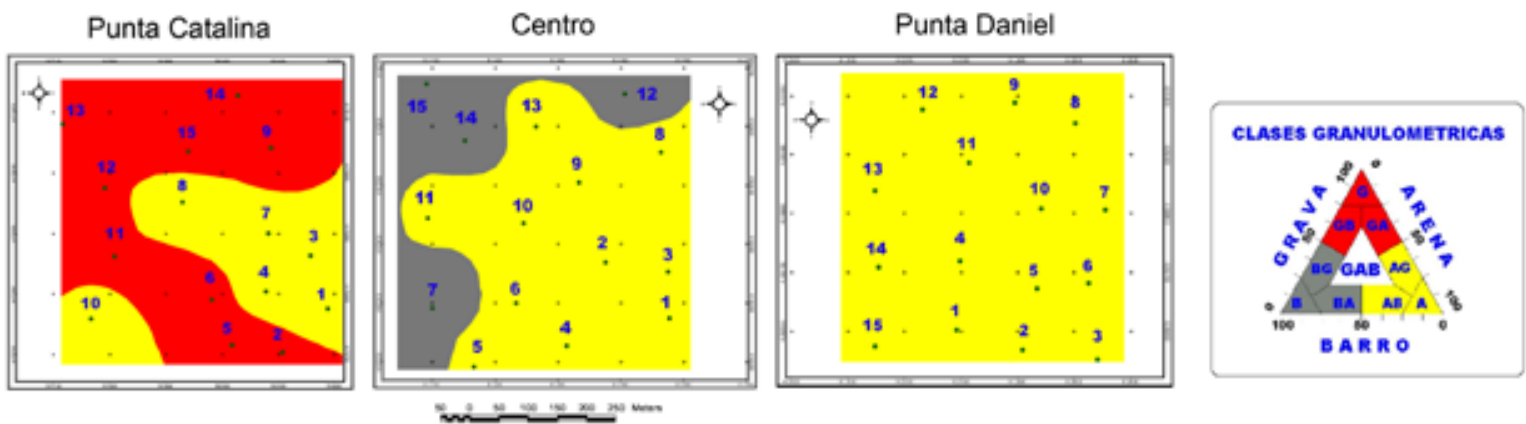

Fig. 2. Clases granulométricas determinadas para los sedimentos superficiales del sublitoral según el diagrama de Shepard (1954), en tres áreas de muestreo en la boca oriental (Atlántica) del estrecho de Magallanes. Los números incluidos en cada plano indican las muestras respectivas recolectadas para el análisis granulométrico. 


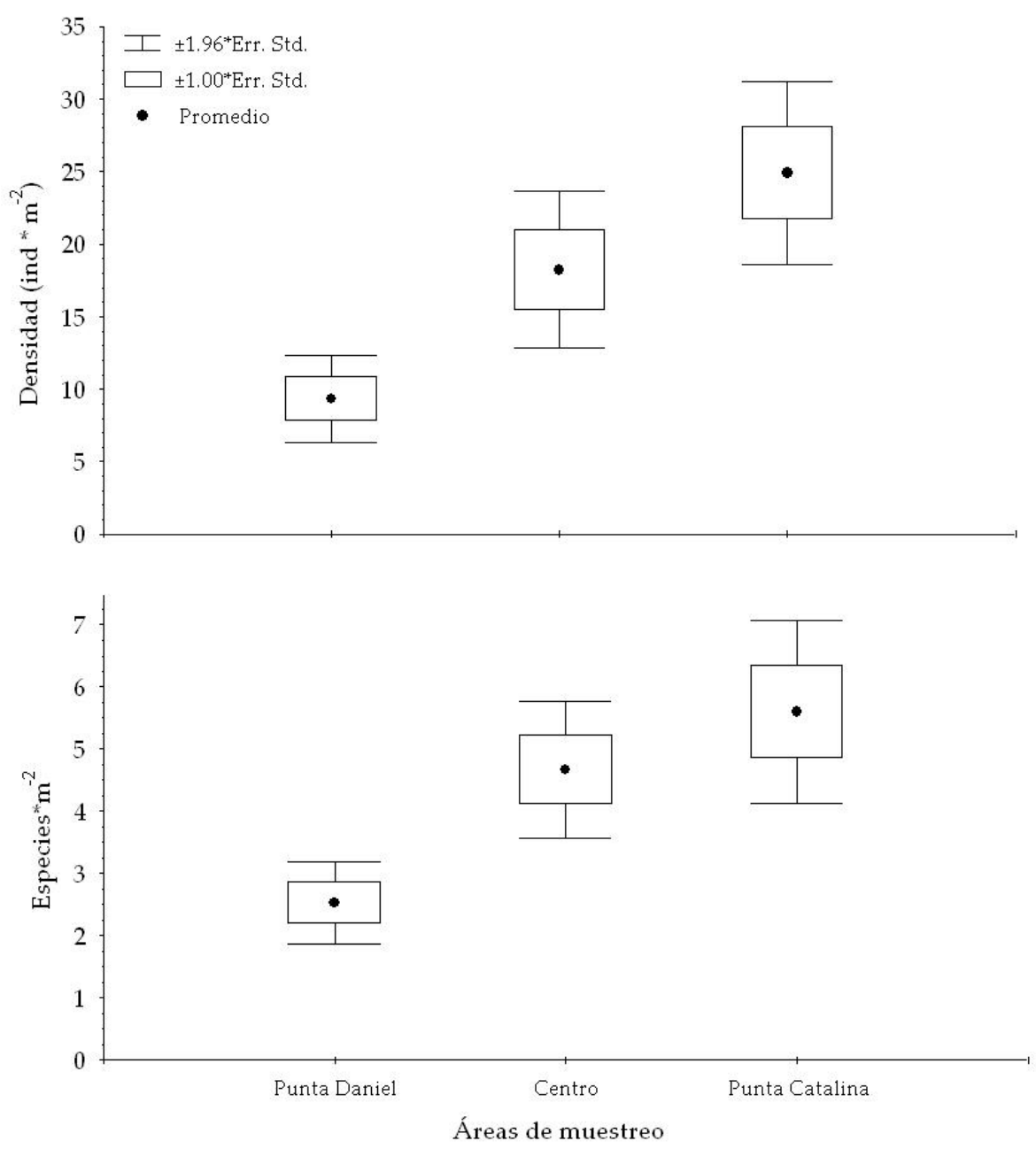

Fig. 3. Densidad (ind $\left./ \mathrm{m}^{2}\right)$ y número promedio de especies $\left(\mathrm{m}^{2}\right)$ del macrobentos sublitoral en tres áreas de muestreo localizadas en la boca oriental (Atlántica) del estrecho de Magallanes.

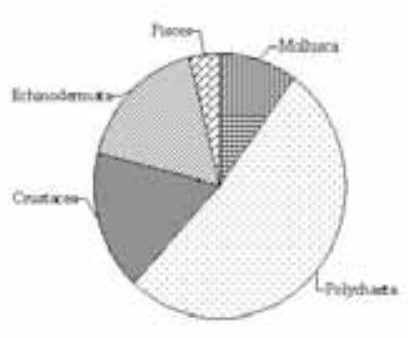

Punta Daniel

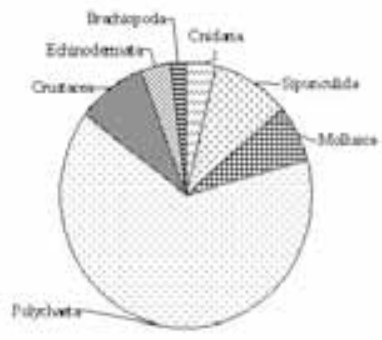

Centro

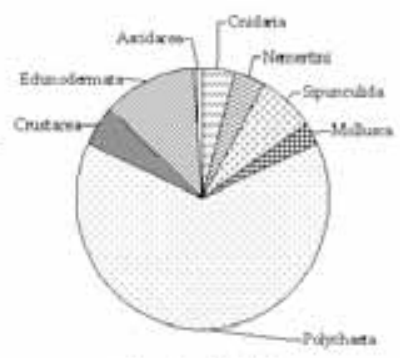

Punta Catalina

Fig. 4. Abundancia relativa del macrobentos sublitoral a nivel de taxa superiores determinados para las tres áreas de muestreo en la boca oriental (Atlántica) del estrecho de Magallanes. 

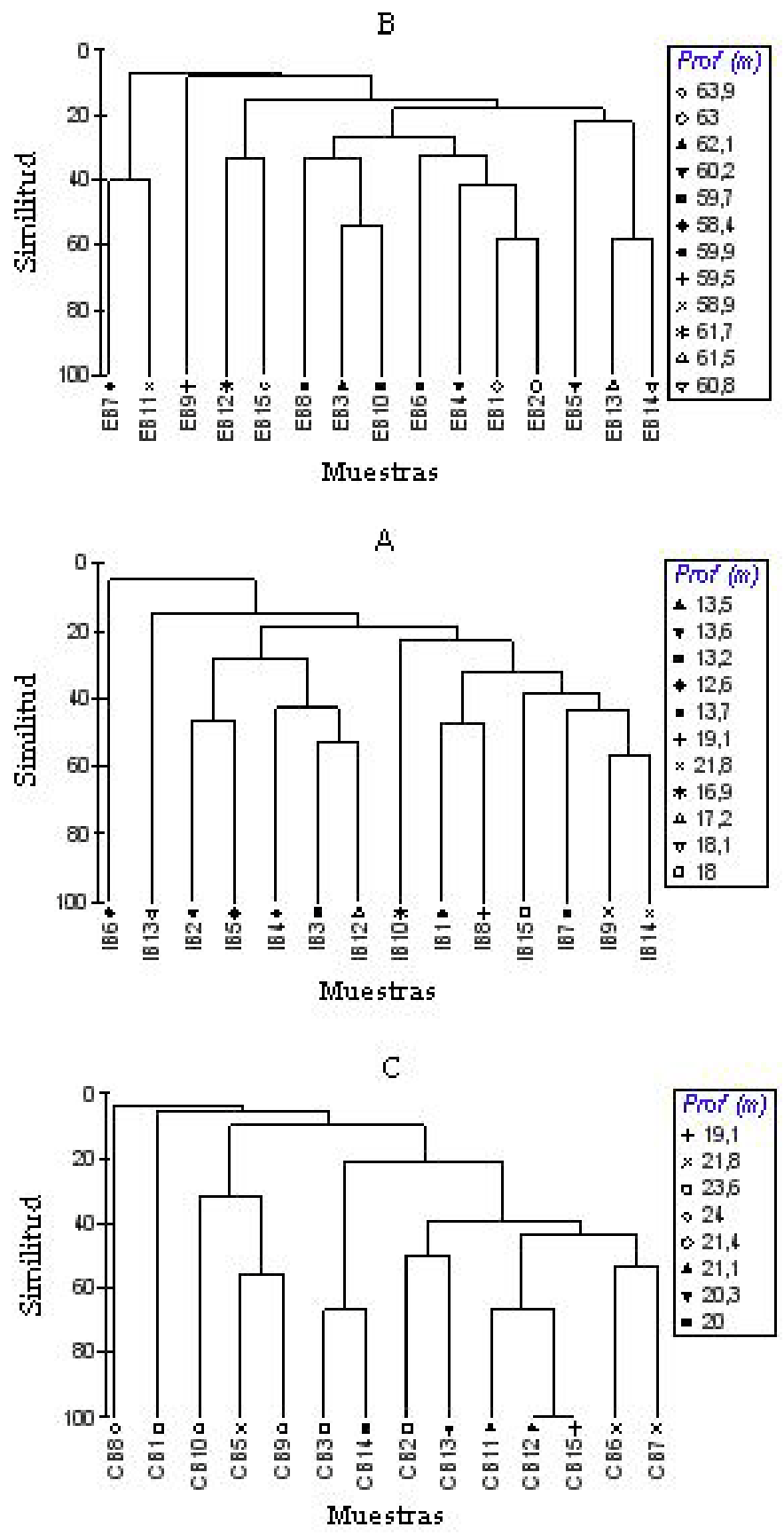

Fig. 5. Dendrogramas para las tres áreas de muestreo $(\mathrm{A}=$ punta Catalina; $\mathrm{B}=$ sector central del estrecho de Magallanes; $\mathrm{C}=$ punta Daniel) producidos con el indice de similitud de Bray-Curtis y con la técnica aglomerativa de agrupamiento promedio. Se incluyen las profundidades (Prof $\mathrm{m}$ ) para cada sitio de muestreo dentro de las tres áreas. 

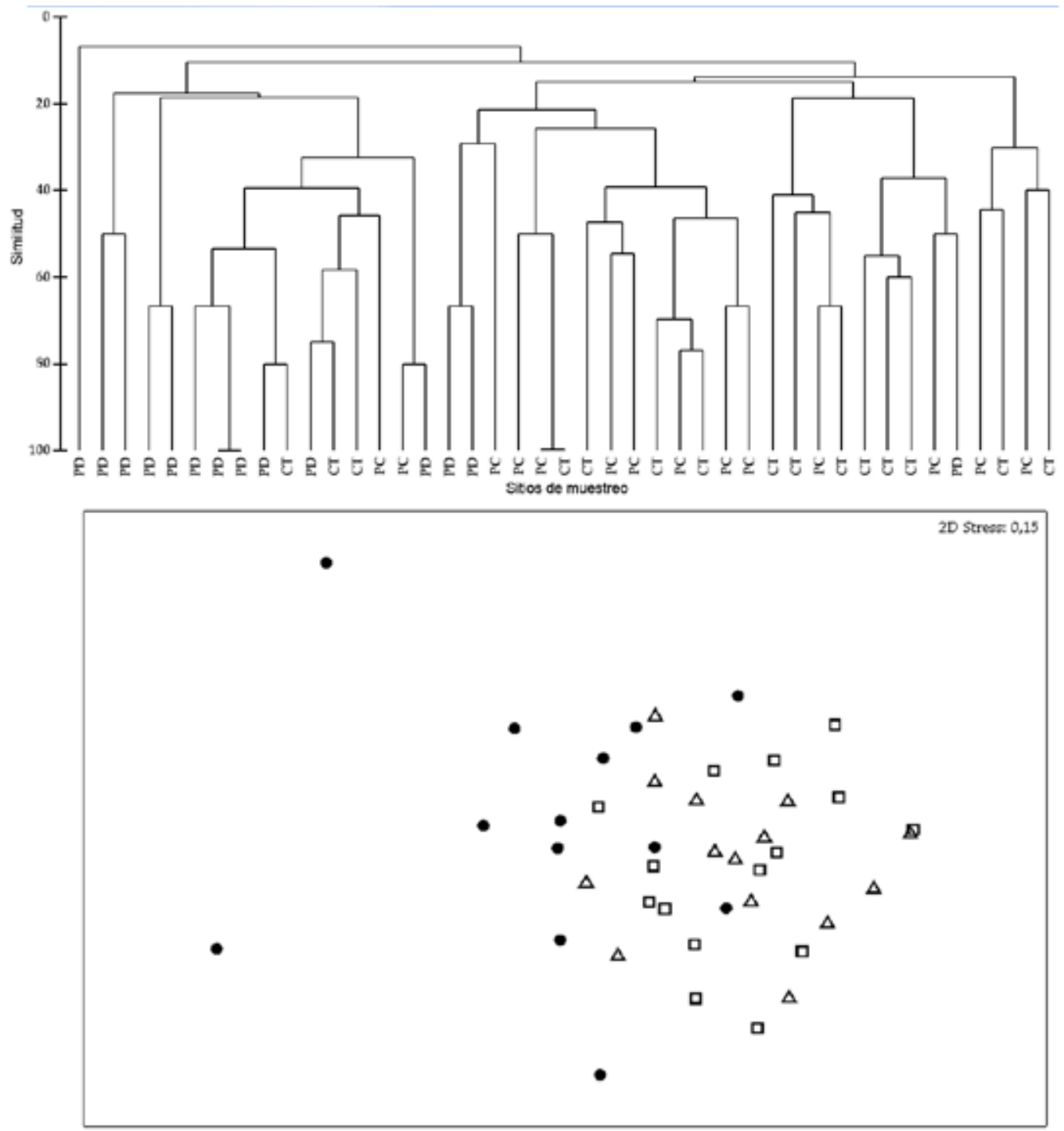

Fig. 6. Dendrograma (A) y ordenamiento multidimensional (MDS; B) para los valores de abundancia promedio (transformación raíz cuarta; Índice de similitud de Bray-Curtis) del macrobentos sublitoral de cada estación de muestreo en la entrada oriental del estrecho de Magallanes. Circulo lleno $=$ punta Daniel (PD en A); triangulo vacío = punta Catalina $(\mathrm{PC}$ en $\mathrm{A})$; cuadrado vacío = sector central $(\mathrm{CT}$ en A). 


\section{AGRADECIMIENTOS}

Agradecemos especialmente a nuestro colaborador Jorge Ramírez V. (Instituto de la Patagonia, Universidad de Magallanes) por su continuo y valioso apoyo en las actividades de terreno y de laboratorio. Asimismo, agradecemos a nuestro colega Ing. Cristian Muñoz (GEA-UMAG) por su importante aporte en los análisis granulométricos y al geógrafo Sr. Daniel-W Antúnez por su aporte en la confección de figuras. Dos revisores anónimos contribuyeron de manera relevante para mejorar el contenido del trabajo. De modo muy especial, agradecemos al revisor $\mathrm{N}^{\circ} 1$ por su acuciosa revisión e interesante aporte de literatura referida al indice de Bray-Curtis.

\section{LITERATURA CITADA}

Antezana, T. 1999. Hydrographic features of Magellan and Fuegian inland passages and adjacent Subantarctic waters. Scientia Marina 63(Suppl. 1): 23-34.

Antezana, T., L. Guglielmo \& E. Ghirardelli 1992. Microbasins within the Strait of Magellan affecting zooplankton distribution. In: Gallardo, V. A., O. Ferretti \& H. I. Moyano (eds.): Oceanografía in Antarctica. pp. 453-458. ENEA-Proyecto Antartica-Italia.

Arntz, W. \& C. Ríos (eds.) 1999. Magellan-Antarctic: Ecosystems that drifted apart. Scientia Marina 63(Suppl. 1): 518 pp.

Bray, J. R. \& J. T. Curtis 1957. An ordination of the upland forest communities of Southern Wisconsin. Ecological Monograph 27: 325-349.

Brambati, A., G. Fontolan \& U. Simeoni 1991. Recent sediments and sedimentological processes in the Strait of Magellan. Bolletino Di Oceanologia Teorica Ed Applicata 9: 217-259.

Clarke, K. R. \& R. N. Gorley 2006. PRIMER v6: User manual/Tutorial. PRIMER-E Ltd. Plymouth Marine Laboratory, U. K. 190 pp.

Escribano, R., M. Fernández \& A. Aranís 2003. Physical-chemical processes and patterns of diversity of the Chilean eastern boundary pelagic and benthic marine ecosystems: an overview. Gayana 67: 190-205.

Fernández, M., E. Jaramillo, P. Marquet, C. Moreno, S. Navarrete, P. Ojeda, C. Valdovinos \& J. Vásquez 2000. Diversity, dynamics and biogeography of Chilean benthic nearshore ecosystem: an overview and guidelines for conservation. $R e-$ vista Chilena de Historia Natural 73: 629-662.
Folk, R. L. 1974. Petrology of sedimentary rocks. Hemphill Publishing Company, Austin, Texas. 182 pp.

Gerdes, D. \& A. Montiel 1999. Distribution patterns of macrozoobenthos: a comparison between the Magellan region and the Weddell Sea (Antarctic). Scientia Marina 63(Suppl. 1): 149-154.

Glorioso, P. D. 1987. Temperature distribution related to the shelf-sea fronts on the Patagonian shelf. Continental Shelf Research 7: 27-34.

Gutt, J., E. Helsen, W. Arntz \& A. Buschmann 1999. Biodiversity and community structure of the mega-epibenthos in the Magellan region (South America). Scientia Marina 63(Suppl. 1): 155-170.

Holte, B. 1998. The macrofauna and main functional interactions in the sill basin sediments of the pristine Holandsfjord, Northern Norway, with autoecological reviews for some key-species. Sarsia 83: 55-68

Kilian, R., O. Baeza, T. Steinke, M. Arevalo, C. Ríos \& C. Schneider 2007. Late Pleistocene to Holocene marine transgression and thermohaline control on sediment transport in the western Magellanes fjord system of Chile (53ㅇ). Quaternary International 161: 90-107.

López-Gappa, J. \& M.C. Sueiro 2007. The subtidal macrobenthic assemblages of Bahía San Sebastián (Tierra del Fuego, Argentina). Polar Biology 30: 679-687.

Lorenti, M. \& S. Mariani 1997. Isopod assemblages in the Straits of Magellan: structural and functional aspects. Polar Biology 18: 254-259.

Magurran, A. E. 2004. Measuring biological diversity. Blackwell Publishing. 256 pp.

Mazzocchi, M. G. \& A. Ianora 1991. A faunistic study of the copepod assemblages in the Strait of Magellan. Bolletino Di Oceanologia Teorica Ed Applicata 9: 163-177.

McCulloch, R. D., C. M. Clapperton, J. Rabasa \& A. P. Currant 1997. The glacial and post-glacial environmental history of Fuego-Patagonia. In: McEwan, C., L. A. Borrero \& A. Prieto (eds.) Patagonia: natural history, prehistory and ethnography at the uttermost end of the Earth. pp. 12-31. British Museum Press, London.

Medeiros, C. \& B. Kjerfve 1988. Tidal characteristics of the Strait of Magellan. Continental Shelf Research 8: 947-960.

Michelato, A., E. Accerboni \& P. Berger 1991. Current meter observations in the eastern and central 
sectors of the Strait of Magellan. Bolletino Di Oceanologia Teorica Ed Applicata 9:261-271. Montiel, A., D. Gerdes \& C. Ríos 2001. Distribución y abundancia del macrozoobentos en una microcuenca marina submareal del estrecho de Magallanes, Chile. Anales Instituto Patagonia (Chile) 29: 117-134.

Montiel, A., D. Gerdes, B. Hilbig \& W. E. Arntz 2005a. Polychaete assemblages on the Magellan and Weddell Sea shelves: comparative ecological evaluation. Marine Ecology Progress Series 297: 189-202.

Montiel, A., D. Gerdes \& W. E. Arntz 2005b. Distributional patterns of shallow-water polychaetes in the Magellan region: a zoogeographical and ecological synopsis. Scientia Marina 69(Suppl. 2): 123-133.

Montiel, A., C. Ríos \& E. Mutschke 2007. Biodiversidad de los poliquetos sublitorales de las costas noroccidental y sur de la isla de Tierra del Fuego (Chile). Anales Instituto Patagonia (Chile) 35(2): 41-52.

Mutschke, E. \& C. Ríos 2006. Distribución espacial y abundancia relativa de equinodermos en el estrecho de Magallanes, Chile. Ciencia y Tecnología del Mar 29(1): 91-102.

Oug, E. 2000. Soft-bottom macrofauna in the high-latitude ecosystem of Balsfjord, northern Norway: Species composition, community structure and temporal variability. Sarsia 85:1-13

Panella, S., A. Michelato, R. Perdicaro, G. Magazzù, F. Decembrini \& P. Scarazzato 1991. A preliminary contribution to understanding the hydrological characteristics of the Strait of Magellan: Austral Spring 1989. Bolletino Di Oceanologia Teorica Ed Applicata 9:107-126.

Ríos, C., E. Mutschke, A. Montiel, D. Gerdes \& W. Arntz 2005. Soft-bottom macrobenthic faunal associations in the southern Chilean glacial fjord complex. Scientia Marina 69(Suppl. 2): 225-236.
Ríos, C., E. Mutschke \& E. Morrison 2003. Biodiversidad bentónica en el estrecho de Magallanes, Chile. Revista de Biología Marina y Oceanografía 38: 1-12.

Salinas, S., M. Contreras \& J. Fierro 2004. Propagación de la onda de marea en el estrecho de Magallanes. Ciencia y Tecnología del Mar 27: 5-20.

Schratzberger, M., N. Lampadariou, P. J. Somerfield, L. Vandepitte \& E. Vanden Berghe 2009. Impact of seabed disturbance on nematode communities: linking field and laboratory observations. Marine Biology 156: 709-724.

Shepard, F. P. 1954. Nomenclature based on sandsilt-clay rations. Journal of Sedimentology and Petrology 24: 151-158.

Sokal, R. R. \& F. J. Rohlf 2003. Biometry. Third edition, eighth printing. W. H. Freeman and Co. 887 pp.

Somerfield, P. J. 2008. Identification of the BrayCurtis similarity index: Comment on Yoshioka (2008). Marine Ecology Progress Series 372: 303-306.

Thatje, S. \& E. Mutschke 1999. Distribution and abundance, biomass, production and productivity of macrobenthos in the Subantarctic Magellan Province (South America). Polar Biology 22: 31-37

Uribe, J. C. 1991. Net-phytoplankton distribution in the Strait of Magellan. Bolletino Di Oceanologia Teorica Ed Applicata 9: 145-150.

Valdovinos, C., S. A. Navarrete \& P. Marquet 2003. Mollusk species diversity in the southern Pacific: why are there more species towards the pole? Ecography 26: 139-144.

Yoshioka, P. M. 2008a. Misidentification of the BrayCurtis similarity index. Marine Ecology Progress Series 368: 309-310.

Yoshioka, P. M. 2008b.The Bray-Curtis similarity index remains misidentified: Reply to Somerfield (2008). Marine Ecology Progress Series 372: 307-309. 